\begin{tabular}{c} 
journal homepage: http://ijiemjournal.uns.ac.rs/ \\
International Journal of Industrial \\
Engineering and Management \\
volume $11 /$ No $1 /$ March $2020 / 40-49$ \\
\hline
\end{tabular}

Original research article

\title{
Mitigating the Impact of Congestion Minimization on Vehicles' Emissions in a Transportation Road Network
}

\author{
S. Salman ${ }^{\mathrm{a}}$, S. Alaswad ${ }^{\mathrm{b}}$ \\ a College of Technological Innovation, Zayed University, Abu Dhabi, UAE; \\ ${ }^{b}$ College of Business, Zayed University, Abu Dhabi, UAE
}

A B STRACT

Traffic optimization normally improves flow conditions at the expense of increased vehicles' emissions. This paper proposes a bi-objective optimization approach to address this situation. In contrast to existing literature, this study considers environmental and congestion impacts of Network Design Problems (NDPs) using the Markov chain traffic assignment approach instead of user equilibrium. The NDP model selectively reverses roads' directions to improve network performance. The model is optimized by simultaneously minimizing maximum traffic density and total vehicles' emissions cost using non-dominated sorting genetic algorithm. A realistic city example was used to demonstrate the approach's efficiency. Results showed that a compromise solution between the two objectives is more practical than single-objective optimization solutions.

Nomenclature

$\begin{array}{llll}\text { BPR } & \text { Bureau of public roads } & \text { CO } & \text { Carbon Monoxide } \\ \text { GA } & \text { Genetic algorithm } & \text { GHG } & \text { Greenhouse gas } \\ \text { MCTA } & \text { Markov chain traffic assignment } & \text { NDP } & \text { Network Design Problem } \\ \text { NO }_{x} & \text { Nitrogen oxides } & \text { TEC } & \text { Total emissions cost } \\ \text { UE } & \text { User equilibrium } & \text { VOC } & \text { Volatile organic compounds }\end{array}$

NSGA-II Non-dominated sorting genetic algorithm
ARTICLE INFO

Article history:

Received September 4, 2019

Revised February 11, 2020

Accepted February 12, 2020

Published online March 3, 2020

Keywords:

Markov chain traffic assignment;

Sustainable network design;

Congestion minimization;

Vehicle emissions;

Bi-objective optimization

*Corresponding author:

Sinan Salman

sinan.salman@zu.ac.ae

\section{Introduction}

In this paper, we study the impact of road congestion minimization on vehicles' emissions. We focus on traffic pattern optimization as a mean for congestion reduction and present an optimization model to help mitigate its environmental effect.

The design of transportation road networks to improve some utility measure is commonly known in the literature as the Network Design Problem (NDP). While the general problem structure remains mostly similar, many approaches have been proposed over the years to handle variations in its modelling and solution methods. Farahani et al. [1] presented a re- view of NDPs in recent literature as part of the larger topic of urban transportation NDPs, which also included public transit network design.

Many NDP approaches found in literature target total travel time as the sole objective for optimization. The underlaying assumption is that minimizing total travel time through network design reduces traffic congestion, which in turn results in lower environmental footprint as smooth traffic flow replaces stopand-go traffic. However, recent literature has been challenging this assumption [2], [3]. In fact, Nagurney showed that a network change resulting in improved travel cost may result in increased total emissions [4]. To understand this paradox in terms of the problem 
presented here (i.e. traffic pattern optimization), consider the following. While network flow improves as traffic restrictions divert vehicles from congested roads, these restrictions also have the side effect of eliminating shorter route options from the network. This translates into longer travel distances for some road users. Consequently, longer travel distances together with increased vehicles' average speeds (due to lower congestion) translate into increased total vehicles' emissions in the network.

As a result, researchers started to include bi-objective modeling in NDPs with increased attention given to environmental impacts [3], [5]. A review of environmental sustainability considerations in road network equilibrium analysis can be found in [6]. The attention to environmental impacts is not surprising given that transportation activities are the top contributor to total greenhouse gas (GHG) emissions, surpassing industrial activities and electricity generation in the USA, as an example [7]. The trend to include environmental impacts in decision making has even made it into corporate strategic agendas [8].

However, most NDP studies still utilize the traditional approach of User Equilibrium (UE) to model traffic assignment and road user behavior. In contrast, this paper explores the use of a recently developed alternative approach, the Markov chain traffic assignment (MCTA), to analyze and mitigate environmental impacts of congestions minimization. MCTA data requirements compared to those of $\mathrm{UE}$ approaches are considerably easier to satisfy. Whereas UE approaches require the creation of an origin-destination demand matrix, which requires conducting road-user surveys followed by a transportation forecasting model to produce the needed data, MCTA requires data that can be collected directly and automatically using sensors installed on the road network. To our knowledge, this study is the first to consider environmental impacts of NDPs using the MCTA approach.

In this paper, we extend the single-objective NDP model proposed in [9] to a bi-objective model that includes network congestion and vehicles' emission objectives. Similar to the single-objective version, the new NDP model selects roads for conversion from two-way traffic to one-way traffic (i.e. traffic direction reversal). This conversion enables significantly more flow capacity in one direction while eliminating traffic flow on the other, for the selected road pair. The goal of these selective road conversions is to improve overall network performance, which we measure here using the network's maximum traffic density and total vehicles' emissions cost.

The approach taken in this study is to analyze the relationship between the two NDP objectives using single-objective and bi-objective optimizations. The single-objective optimization was deployed to study the impact of congestion optimization on emissions. To accomplish this, the NDP was optimized using maximum traffic density while at the same time total vehicles' emissions cost passively tracked. Due to problem complexity and large solution space, we used a Genetic Algorithm (GA) to solve the NDP. Next we deployed bi-objective optimization to define a pareto-front which was then used to find compromise solutions, mitigating the impact of congestion minimization on the network's environmental footprint. Similarly, due to problem complexity and its large solution space, we used the well-known fast non-dominated sorting genetic algorithm (NSGA-II) [10] in solving the bi-objective NDP.

The remainder of this paper is organized as follows. In section 2, we briefly introduce MCTA theory, and the NDP formulation including total cost of vehicles' emissions as a second objective. In sections 3 , we present the bi-objective optimization solution method and investigate its results for a real city example. Finally, we conclude and point to future work in section 4 .

\section{Modelling Network Design Problems using Markov Chain Traffic Assignment}

\subsection{An Overview of Markov Chain Traffic Assignment}

The application of Markov chain analysis to transportation road networks and traffic assignment is not new. Crisostomi et al. [11] were the first to thoroughly define and analyze the application of this approach mathematically. The following is a summary of their approach. Throughout this paper we use "road" to describe a line segment between two points on a network where a vehicle must travers the entire line before it can transition into another. Using this definition, streets with intermediate entry or exit points are simply segmented into two (or more) roads meeting at such points.

In the MCTA approach, each road is modelled as a state in a Markov chain. Vehicles transition from one state (road i) to another (road j) with a transition probability $\dot{\mathrm{p}}_{\mathrm{ij}}$. This probability can be derived from network sensors counting vehicles transitioning between roads at intersections and entry/exit points. If a transition between two roads is not possible (i.e. 
roads are not directly connected or turning is prohibited), the transition probability between the two roads is set to zero. The result is a sparse transition probability matrix, which we refer to as Ṕ.

Since roads in a network have different lengths, the probability of a vehicle staying on a given road rather than transitioning to the next step in its journey depends on the vehicle's travel time $t_{i}$ for that road. In turn, a vehicle's travel time for a road depends on the road's length and the vehicle's average speed on that road. Modifying transition probabilities using (1) and (2) incorporates this into a modified $\mathrm{P}$ matrix for use in the MCTA model:

$$
\begin{aligned}
& p_{i i}=\frac{t_{i}-1}{t_{i}}, i=1, \ldots, n \\
& p_{i j}=\left(1-p_{i j}\right) \dot{p}_{i j}, i \neq j
\end{aligned}
$$

where $\mathrm{n}$ is the number of roads in the network. To complete the analysis, we define $\pi$ as the stationary distribution vector, whose elements $\pi_{\mathrm{i}}$ represent the probabilities of a vehicle being on road $i$ in a network operating in steady state. Markov chain theory states that stationary state probabilities can be obtained via a unique solution $(\pi)$ to the matrix-form system of equations represented in (3), and that it exists only if the chain is irreducible and aperiodic [12]. In transportation networks, both conditions hold true.

$$
\pi \mathrm{P}=\pi
$$

After solving for $\pi$, and given an estimate of the total number of vehicles $\mathrm{V}$ traversing the network at the time of data collection, we can calculate the expected number of vehicles on each road in the network using $\mathrm{V} \pi_{\mathrm{i}}$. These expected values represent the traffic assignment of vehicles to roads in a given road network at a given traffic state. This application of Markov chain theory to traffic assignment is used in several other literature studies [9], [13]-[17].

\subsection{Modeling the Network Design Problem}

\subsubsection{Single Objective Model: Maximum Traffic Density}

The NDP model summarized in this section is the single objective model proposed in [9]. We extend this model into a bi-objective NDP model in the following section.

We start by defining the matrix of decision variables $X_{d}$, which is a diagonal matrix with binary deci- sion variable $\mathrm{X}_{\mathrm{d}_{\mathrm{i}}}$ representing the decision to reverse road i's traffic flow $\left(X_{d_{i}}=0\right)$ or to leave it unaltered $\left(X_{d_{i}}=1\right)$. All non-diagonal elements in $X_{d}$ are zeros by definition. In addition, we define the following sets of constants for the modeled network: $\mathrm{N}_{\mathrm{i}}$ is the number of lanes in road $\mathrm{i}, \mathrm{L}_{\mathrm{i}}$ is the length of road $\mathrm{i}$, and $\mathrm{r}_{\mathrm{i}}$ is the index of the road that is parallel but opposite in direction to road i. To illustrate the use of $r_{i}$, consider the hypothetical example of Elm street which runs east-west; we can designate the eastbound $\operatorname{road}$ as $i=1$, the westbound road as $i=2$, and the corresponding opposite direction road indices of $r_{1}=2$ and $r_{2}=1$. We refer to the two roads as opposing direction roads. This allow us to model the relationship between the two sides of Elm street. An example illustration of a road network described using the terms defined here can be found in Section 3 (see Figure 5). The figure shows network traffic modifications applied to the City of Abu Dhabi.

Next, we describe the model presented in (4) through (10) in reverse order starting with Constraint (10), which ensures that any two opposing direction roads can at most have one of the sides reversed; either $\mathrm{X}_{\mathrm{d}_{\mathrm{i}}}=0$ or $\mathrm{X}_{\mathrm{d}_{\mathrm{r}_{\mathrm{i}}}}=0$, but not both. Constraint (9) reassigns the number of lanes from the reversed road to its opposing direction road effectively, closing the former and increasing the capacity of the latter. The resulting variable $\hat{\mathrm{N}}_{\mathrm{i}}$ indicates the modified road capacity (i.e. number of lanes). Constraint (7) modifies the transition probability matrix $\mathbf{P}$ by eliminating all transition probabilities for reversed flow roads (via $\mathrm{X}_{\mathrm{d}} \mathrm{PX}_{\mathrm{d}}$ ), while maintaining the stochastic property of the resulting matrix (via multiplying by $S_{d}$ ). The factor diagonal matrix $S_{\mathrm{d}}$ is defined using Constraint (8). The result is a modified network transition probability matrix $\hat{P}$. Constraint (6) produces the stationary distribution vector, and Constraint (5) defines the networks' maximum road traffic density value, which is the objective of this NDP model.

$$
\text { Minimize } \mathrm{D}_{\max }
$$

Subject to:

$$
\frac{\mathrm{V} \pi_{\mathrm{i}}}{\mathrm{L}_{\mathrm{i}} \hat{\mathrm{N}}_{\mathrm{i}}} \leq \mathrm{D}_{\max }
$$

$$
\begin{aligned}
& \pi \hat{\mathrm{P}}=\pi \\
& \hat{\mathrm{P}}=\mathrm{S}_{\mathrm{d}} \mathrm{X}_{\mathrm{d}} \mathrm{PX}_{\mathrm{d}}
\end{aligned}
$$




$$
\begin{aligned}
& \mathrm{S}_{\mathrm{d}_{\mathrm{i}}}=\left\{\begin{array}{cl}
\frac{1}{\sum_{\mathrm{j}=1}^{\mathrm{n}} \mathrm{P}_{\mathrm{ij}} X_{\mathrm{dj}_{j}}}, & \text { if } \mathrm{X}_{\mathrm{d}_{\mathrm{i}}}=1 \\
1, & \text { if } \mathrm{X}_{\mathrm{di}_{\mathrm{i}}}=0
\end{array}\right. \\
& \hat{N}_{d}=X_{d_{i}} N_{i}+\left(1-X_{d_{r_{i}}}\right) N_{r_{i}} \\
& \mathrm{X}_{\mathrm{d}_{\mathrm{i}}}+\mathrm{X}_{\mathrm{d}_{\mathrm{r}_{\mathrm{i}}}} \geq 1 \\
& \text { Where } \quad \mathrm{X}_{\mathrm{d}_{\mathrm{i}}} \in\{0,1\} \text {, } \\
& \pi_{\mathrm{i}} \geq 0 \text {, } \\
& |\pi|=1 \text {, } \\
& \mathrm{i}=1, \ldots, \mathrm{n} \text {, } \\
& \mathrm{j}=1, \ldots, \mathrm{n} \text {. }
\end{aligned}
$$

The resulting $\mathrm{D}_{\max }$ value can be compared to level of service (LoS) values published in the Highway Capacity Manual [18]. For example, a traffic density higher than 28 vehicles $/ \mathrm{km}$ - lane indicates a breakdown in traffic conditions resulting in unstable network flow.

\subsubsection{Bi-Objective Model: Accounting for Environmental Impact via Total Vehicles' Emissions Cost}

There are several methods used in practice as well as in research for estimating vehicle's emissions. The methods vary widely depending on available input data, method complexity, and desired output detail-level. Readers are referred to Smit [19] for a review of the different methods used for emissions estimation.

MCTA is a macroscopic transportation model that represents vehicles' collective behavior in a network, rather than an individual vehicle's behavior as represented in microscopic models. This means that vehicle instantaneous operating conditions such as instantaneous fuel consumption, vehicle acceleration, and engine rotational speed at various points of the vehicle's journeys are not available. However, traffic congestion conditions at any given road in a network can be easily produced using MCTA's out-

Table 1. TRANSYT-7F coefficients and monetization factors* put via traffic density calculated at road level. The macroscopic property of the MCTA model narrows down the categories of emissions estimation methods that can be utilized in this application.

Vehicle emissions estimation in macroscopic transportation models is typically carried out using a category of estimation methods known as average speed emission models. In this paper, we utilize the widely cited TRANSYT-7F estimation model [20], which was used in many literature studies [21]-[25]. Equation (11) shows the TRANSYT-7F general formula for road i and pollutant p. Values for the coefficients $A_{p}, B_{p}$, and $C_{p}$ for each pollutant used in this study are listed in Table 1 . Here, $\mathrm{S}_{\mathrm{i}}$ is the average vehicles speed on road i measured in $\mathrm{ft} / \mathrm{s}$. The resulting estimated emission $Q_{i}{ }^{p}$ is expressed in $g / f t . v e h$, which requires conversion to $\mathrm{kg} / \mathrm{km} \cdot$ veh for use in subsequent steps.

$$
Q_{i}^{p}=\frac{A^{p} \cdot e^{\left(B^{p} \cdot S_{0}\right)}}{C^{p} \cdot S_{i}}
$$

Since there are several GHGs resulting from fuel combustion in vehicles, we focus in this study on the most commonly reported GHG in similar studies: carbon monoxide $(\mathrm{CO})$, nitrogen oxides $\left(\mathrm{NO}_{\mathrm{X}}\right)$, and volatile organic compounds (VOC). These three emissions are typically selected due to their high share of total vehicular emissions and significant impact on human health. We also combine the impact of the three selected emissions into a single cost measure using monetization factors deduced from [26], after adjusting for inflation; see $\mathbf{M}^{\mathrm{p}}$ in Table 1 . A similar approach was taken by Szeto et al. [27] and Wang and Szeto [5]. Total road emissions cost $\mathrm{EC}_{\mathrm{i}}$ for road i can be calculated via (12), expressed in $\$ /$ veh. Road

\begin{tabular}{|c|c|c|c|c|}
\hline Pollutants & $A^{\mathrm{P}}(\mathrm{g} / \mathrm{ft} \cdot \mathrm{veh})$ & $B^{P}(s / f t)$ & $C^{p}(s / f t)$ & $\mathrm{M}^{\mathrm{P}}(\$ / \mathrm{kg})$ \\
\hline $\mathrm{CO}$ & 3.3963 & 0.014561 & 1,000 & 0.93070 \\
\hline $\mathrm{NO}_{\mathrm{x}}$ & 1.5718 & 0.040732 & 10,000 & 1.89719 \\
\hline VOC & 2.7843 & 0.015062 & 10,000 & 2.50572 \\
\hline
\end{tabular}
emissions can be summarized on a network level by multiplying $\mathrm{EC}_{\mathrm{i}}$ by the road's vehicle flow rate $\mathrm{v}_{\mathrm{i}}$, which in turn can be calculated via the fundamental flow relation $\mathrm{v}_{\mathrm{i}}=\mathrm{D}_{\mathrm{i}} \cdot \mathrm{S}_{\mathrm{i}}$, and then totaling all roads' emissions costs. Equation (13) shows the final formula for total network emissions costs (TEC), expressed in $\$ / \mathrm{hr}$. In the equation, $\mathrm{D}_{\mathrm{i}}$ is the traffic density resulting from vehicles using road $i$.

*partially adapted from [5] and [26] 


$$
\begin{aligned}
& \mathrm{EC}_{\mathrm{i}}=\Sigma_{\mathrm{p}} \mathrm{Q}_{\mathrm{i}}^{\mathrm{p}} \cdot \mathrm{L}_{\mathrm{i}} \cdot \mathrm{M}^{\mathrm{p}}, \forall \mathrm{p} \\
& \mathrm{TEC}=\Sigma_{\mathrm{i}} \mathrm{EC}_{\mathrm{i}} \cdot \mathrm{D}_{\mathrm{i}} \cdot \mathrm{S}_{\mathrm{i}}, \forall \mathrm{p}
\end{aligned}
$$

A closer look at Equations (11), (12), and (13) reveals that all terms used in calculating TEC are constants except for $D_{i}$ and $S_{i}$, and while $D_{i}$ is a direct result of MCTA, $\mathrm{D}_{\mathrm{i}}$ is not and requires estimation based on traffic conditions associated with $\mathrm{D}_{\mathrm{i}}$. To achieve this, we utilized the well-known Bureau of Public Roads (BPR) formula shown in (14). The formula defines a relationship between flow rate and travel time as experienced by vehicles using the road, given its maximum flow capacity $\mathrm{c}$ and free flow travel time tf. The Highway Capacity Manual [18] lists different $\mathrm{c}$ values depending on road type and free flow speed. Similarly, a and b are constants determined by road type but are often deployed in literature with the generalized values of $\mathrm{a}=0.15$ and $\mathrm{b}=4$.

$$
\mathrm{t}=\mathrm{t}_{\mathrm{f}}\left(1+\mathrm{a}\left(\frac{\mathrm{v}}{\mathrm{c}}\right)^{\mathrm{b}}\right)
$$

It is possible to use the fundamental flow relation and the BPR formula to derive a relationship between traffic density $\mathrm{D}$ and vehicle speed $\mathrm{S}$. This results in the polynomial relationship defined in (15), where $S_{\mathrm{f}}$ is the average vehicles speed in free flow conditions, and $\mathrm{D}_{\mathrm{c}}$ is traffic density at road's capacity (when $\mathrm{v}=\mathrm{c}$ ). However, deriving a closed form general solution to this polynomial relationship is not possible due to the resulting quintic polynomial function.

$$
\frac{1}{\mathrm{~S}^{\mathrm{b}+1}}-\frac{1}{\mathrm{~S}_{\mathrm{f}} \cdot \mathrm{S}^{\mathrm{b}}}-\mathrm{a}(1+\mathrm{a})^{\mathrm{b}}\left(\frac{\mathrm{D}}{\mathrm{D}_{\mathrm{c}}}\right)^{\mathrm{b}} \frac{1}{\mathrm{~S}_{\mathrm{f}}^{\mathrm{b}+\mathrm{I}}}=0
$$

We overcame this obstacle by evaluating the BPR formula at many $\mathrm{v}$ values covering the range of possible flow values needed in the optimization model. The resulting travel times at various flow values were used to calculate corresponding $\mathrm{D}$ and $\mathrm{S}$ values.
These corresponding values were then used to define a speed estimation function $S \cong f\left(D, S_{f}\right)$ where interpolation was used to fill-in any missing values in the relationship. Figure 1 shows the resulting relationship for four types of roads defined by their free flow speeds $S_{\mathrm{f}}: 60,80,100$, and $120 \mathrm{~km} / \mathrm{h}$.

Finally, we inserted the TEC objective into the NDP model as a second objective. Its insertion did not introduce any additional constraints over the single-objective NDP model, however, it resulted in the inclusion of the speed estimation function described above, as shown in (18). The NSGA-II solution method described in the following section is well suited for handling such complexity. The resulting bi-objective NDP model is as follows:

Minimize $\mathrm{D}_{\max }$ and TEC

$$
\begin{aligned}
& \text { Subject to Constraints (5) } \\
& \text { trough (10) }
\end{aligned}
$$

$$
\text { Where } S_{\mathrm{i}} \cong \mathrm{f}\left(\mathrm{D}_{\mathrm{i}}, \mathrm{S}_{\mathrm{f}}\right)
$$

\section{Solving the Network Design Problem}

The NDP model presented in section 2.2.2 includes several complexities that reduce the choice of solution methods capable of handling their requirements. These complexities include binary decision variables, nonlinearity, and the use of a piece-wise approximation function. In addition, the problem's solution space $\left(3^{\mathrm{n} / 2}\right.$ where $\mathrm{n}$ is the number roads in a network) is quite large even for medium sized cities when modeling only arterial roads.

For these reasons, we selected GA and NSGA-II as solution approaches for the single- and bi-objective NDP models, respectively. NSGA-II is a GA with a



Figure 1. Vehicles' average speed vs. road density relationship 
fast non-dominant sorting algorithm of fitness values enabling it to handle multi-objective optimization problems similar to the one at hand. The reader is referred to Konak et al. [28] for a good tutorial on multi-objective GA optimization.

The solution encoding and general heuristic setup deployed here, which we summarize below, are similar to those used in [9]. Each gene in the solution encoding represents a pair of opposing direction roads. The gene can indicate one of three states: reversed flow direction in the first road, reversed flow direction in the second road, or both roads have unaltered flow direction. Here, the firstroad refers to the road with lower index value of the pair. In this approach, a solution is simply a sequence of genes representing direction reversal states of all roads in the network. The encoding scheme deliberately excludes the case in which both roads are revered in traffic direction, as such cases do not change network traffic. It also reduces solution space significantly (from $2^{\text {n }}$ to $3^{\text {n/2 }}$ ) and eliminates the need for the constraint listed in (10) in the optimization model due to the use of road pairs in its gene representation instead of individual roads.

The solution approach utilized standard crossover and mutation GA operators. The crossover operator was designed to randomly select two solutions and two crossover points on their gene sequence and swap genes between the two points across the two solutions. Similarly, the mutation operator was designed to randomly select a solution and then a gene within its sequence to be randomly mutated to one of the two other possible states. The remaining GA setup parameters wereset as follows: population size of 192, top 56 solutions kept between generations (about $29 \%$ of population), 50\% probability of deploying crossover, and $18 \%$ probability of deploying mutation. Furthermore, the starting population was initialized to include 182 random solutions and
10 solutions which represented the existing network state (approximately $95 \%$ and $5 \%$ of the population, respectively). A stopping criteria of 1000 generations was used.

The Abu Dhabi island road network was used to demonstrate the application of the proposed approach to a realistic city scenario. The selected area includes 360 arterial road segments covering the island portion of the city. The city's road network data, such as network structure, number of lanes, and speed limits were obtained from OpenStreetMap. org. To facilitate the scenario, turning probabilities were set as follows: U-turns $5 \%$, left-turn $10 \%$, rightturn $10 \%$, and forward $75 \%$. Turn probabilities were adjusted to maintain proportionality where certain turns are not permitted by network design or road nature.

To illustrate the advantage of optimizing NDP using bi-objective optimization rather than single objective optimization, we first applied the single-objective GA approach to the Abu Dhabi road network using the model described in section 2.2.1. While $\mathrm{D}_{\max }$ was the objective used in optimization, the algorithm was modified to passively report total emissions cost for every generation best solution $\left(\mathrm{TEC}^{\mathrm{D}_{\max }}\right)$ with no impact to the optimization process. For this experiment, we used an estimated 15,000 vehicles traversing the city road network to illustrate the optimization impact on a highly congested network.

Figure 2 shows that although the GA progressively produced lower congestion network setups $\left(\mathrm{D}_{\max }\right.$ : $51.23 \rightarrow 31.96$ vehicles $/ \mathrm{km} \cdot$ lane, or $37.6 \%$ reduction), the produced solutions ultimately led to higher total network emissions $\left(\right.$ TEC $^{\mathrm{D}_{\text {max }}}$ : $\$ 213.06 / \mathrm{hr} \rightarrow$ $\$ 215.01 / \mathrm{hr}$, or $0.9 \%$ increase). While the increase in total emissions cost may not seem significant, it is an hourly rate where the difference in emissions accumulates over long periods of time exasperating an undesirable environmental trend. This finding sup-
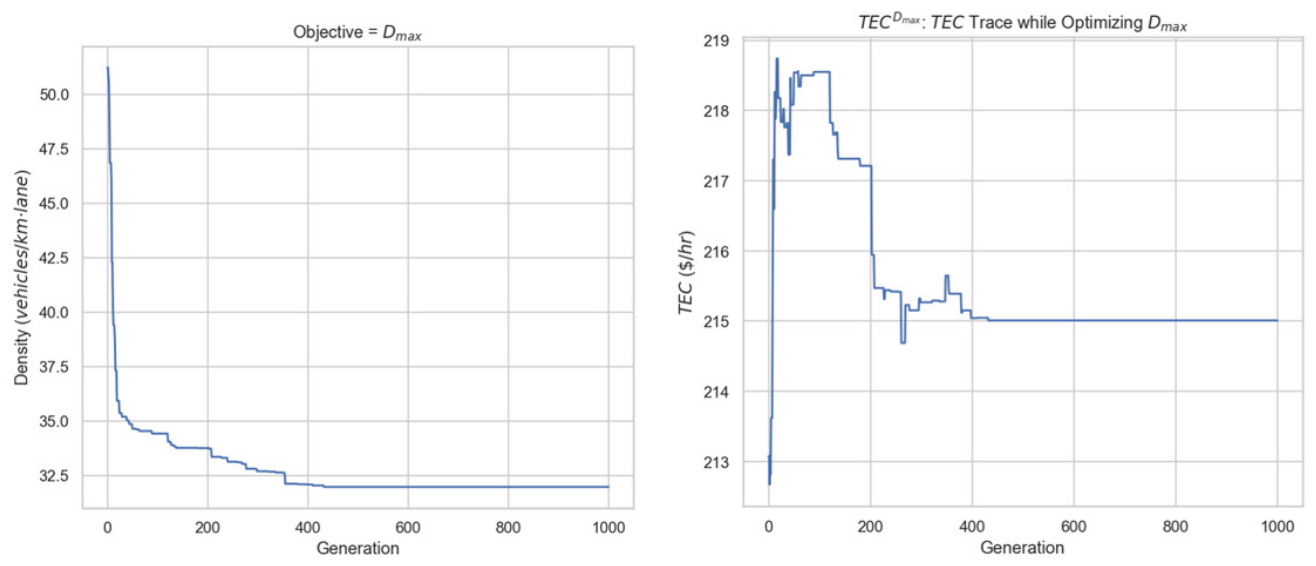

Figure 2. Single objective GA optimization results: minimizing $D_{\max }$, while tracing its impact on TEC 
ports recent literature indicating that the relationship between the two objective functions is generally an inverse relationship, where optimizing network design for congestion minimization often adversely impacts total vehicles' emissions[2]-[4].

In comparison, optimizing the same network using bi-objective NSGA-II produced the results shown in Figure 3. While the final generation solution produced substantially lower network emissions (TEC: $\$ 213.06 / \mathrm{hr} \rightarrow \$ 136.78 / \mathrm{hr}$, or $35.8 \%$ reduction), it also resulted in increased network congestion in comparison to the initial network state $\left(\mathrm{D}^{\max }: 51.23\right.$ $\rightarrow 55.26$ vehicles $/ \mathrm{km} \cdot$ lane, or $7.9 \%$ increase). This renders the final solution undesirable.

On the other hand, solutions found between generations 600 and 700 of the GArun produced both lower network congestion $\left(\mathrm{D}_{\max }=40.99\right.$ vehicles/ $\mathrm{km} \cdot$ lane, or $20 \%$ reduction) and emissions (TEC= $\$ 189.27 / \mathrm{hr}$, or $11 \%$ reduction) compared to the initial network state.

Plotting the pareto-front of non-dominated solutions found throughout the GA run provides a clearer view on the inverse relationship between $\mathrm{D}_{\max }$ and TEC. Figure 4 shows the pareto-front and indicates the current network state (highlighted using a red

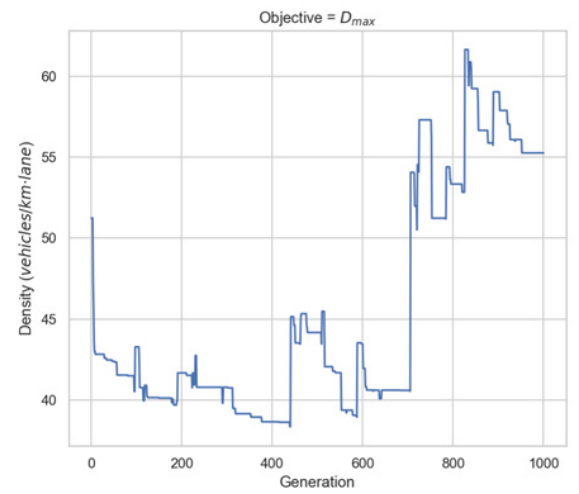

marker). The curve also serves as a decision support tool for decision makers to select compromise solutions that fit different network scenarios and urban planning needs. For example, the compromise solution at generation 650 is highlighted using green marker in Figure 4.

While NDP literature includes several studies with multi-objective models, to our knowledge, there are no studies that use the MCTA approach to optimize models with emissions and congestion objectives. Nevertheless, we compared general trends identified here to that of similar studies to validate our findings. In contrast to MCTA, UE is frequently used with a total system travel time (TSTT) objective. Realizing that both $\mathrm{D}_{\max }$ and TSTT are used as indicators of network congestion, trends between each of them and total emissions should be directionally comparable. To that end, we compared our emissions to congestion trend (i.e. TEC vs. $\mathrm{D}_{\max }$ in Figure 4) to those reported in the literature (emissions vs. TSTT) [5], [23]. The comparison revealed a similar inverse and nonlinear relationship, however differing in shape and scale due to the differing measurements used (density vs. TSTT and emissions mass vs. emissions cost).

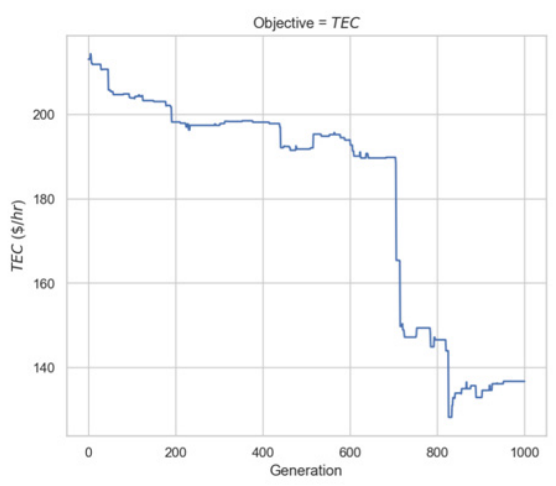

Figure 3. Bi-objective GA optimization results: minimizing $D_{\max }$ and TEC

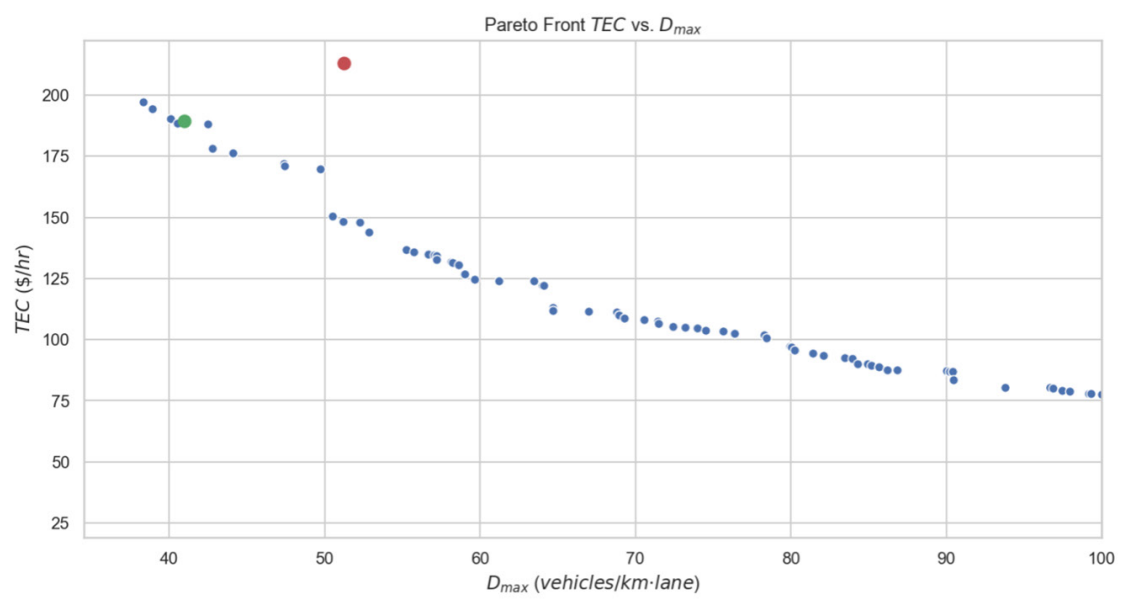

Figure 4. Pareto-front for non-dominated solutions generated in bi-objective NSGA-II (red marker indicates current network state, and green marker indicates compromise solution at generation 650) 
Plotting the pareto-front of non-dominated solutions found throughout the GA run provides a clearer view on the inverse relationship between $\mathrm{D}_{\max }$ and TEC. Figure 4 shows the pareto-front and indicates the current network state (highlighted using a red marker). The curve also serves as a decision support tool for decision makers to select compromise solutions that fit different network scenarios and urban planning needs. For example, the compromise solution at generation 650 is highlighted using green marker in Figure 4.

While NDP literature includes several studies with multi-objective models, to our knowledge, there are no studies that use the MCTA approach to optimize models with emissions and congestion objectives. Nevertheless, we compared general trends identified here to that of similar studies to validate our findings. In contrast to MCTA, UE is frequently used with a total system travel time (TSTT) objective. Realizing that both $\mathrm{D}_{\max }$ and TSTT are used as indicators of network congestion, trends between each of them and total emissions should be directionally comparable. To that end, we compared our emissions to congestion trend (i.e. TEC vs. $\mathrm{D}_{\max }$ in Figure 4) to those reported in the literature (emissions vs. TSTT) [5], [23]. The comparison revealed a similar inverse and nonlinear relationship, however differing in shape and scale due to the differing measurements used (density vs. TSTT and emissions mass vs. emissions cost).

The compromise solution is illustrated in Figure 5 , where 34 out of the 360 network roads were identified by the optimization for traffic flow reversal. The affected roads are highlighted in red. The effects of this solution on network's congestion and emissions are illustrated in Figures 6 and 7 below. Figure 6 shows that the solution reduces congestion in roads in the western outer rim of the city road network.
This was accomplished through redistribution of traffic density on the less trafficked parts of the network, while still maintaining lower congestion levels (i.e. $\mathrm{D}_{\max }$ value). Figure 7 illustrates the emissions impact of the solution, where TEC reduced for some of the most polluted roads and the network as a whole.

The GA and NSGA-II optimization consumed 84 and 87 minutes, respectively, on an Intel Core i7 (i7-6700K) processor taking advantage of all cores. Both heuristics were coded using Python and utilized the DEAP evolutionary computational framework [29] and Python's scientific environment library SciPy [30].

\section{Conclusion}

In this paper, we proposed a bi-objective NDP model utilizing Markov chains for traffic assignment. The model incorporated traffic congestion and environmental objectives to help decision makers understand and leverage the trade-offs involved in traffic pattern optimization. While recent literature pointed to the inverse relationship between traffic congestion and vehicle emissions, the contribution of this work laid in the application of the MCTA modeling approach to demonstrate this phenomenon. It also provided a decision support tool to help traffic planners optimize traffic patterns while taking into consideration the environmental impact of their decision. The reduced data requirements and the relatively short run time required for modeling a real-size city scenario using this approach is conducive to its adoption in practice.

In particular, the Pareto-Front results demonstrated the inverse relationship between network congestion measured via traffic density and total network emissions costs. While current network performance

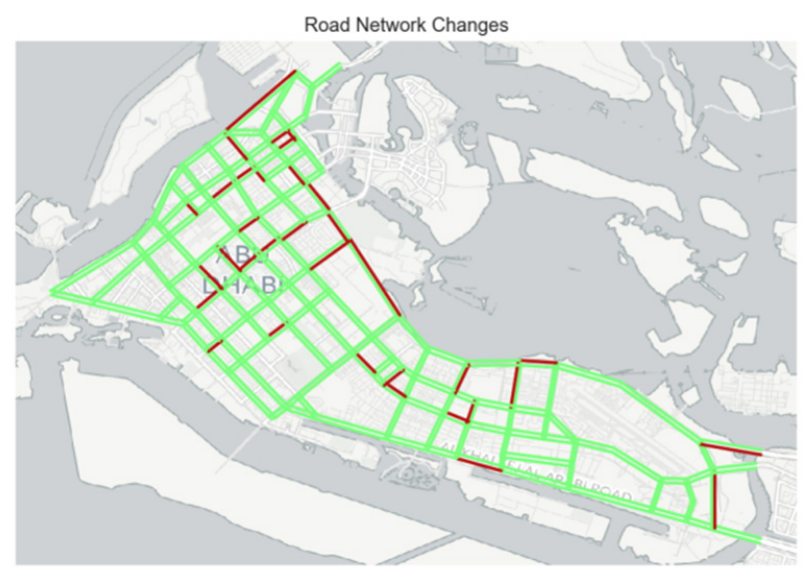

Figure 5. Network modifications for the compromise solution; road direction reversals highlighted in red 
can be improved upon in both objectives (see Figure 4; green marker solution; $7.9 \%$ and $11 \%$ reductions in $\mathrm{D}_{\max }$ and TEC, respectively), it is clear that a criterion must be set by decision makers for prioritizing network congestion and emissions objectives to achieve better overall network traffic performance. Relying on the traditional approach of minimizing congestion only in the presented example resulted in $37.6 \%$ reduction in congestion, however at the cost of increased emissions (0.9\% TEC increase). One possible approach would be to select solutions that minimize congestion without adversely impacting network emissions or setting bounds on acceptable emission levels to find best congestion traffic patterns based on these bounds.

The presented approach can be extended to include multi-objective optimization by adding an objective function to measure network's service level to road users. In addition, new heuristics such as the bee colony optimization and the chemical reaction optimization may provide improved solution searchperformance. These extension ideas are left as future directions for this work.
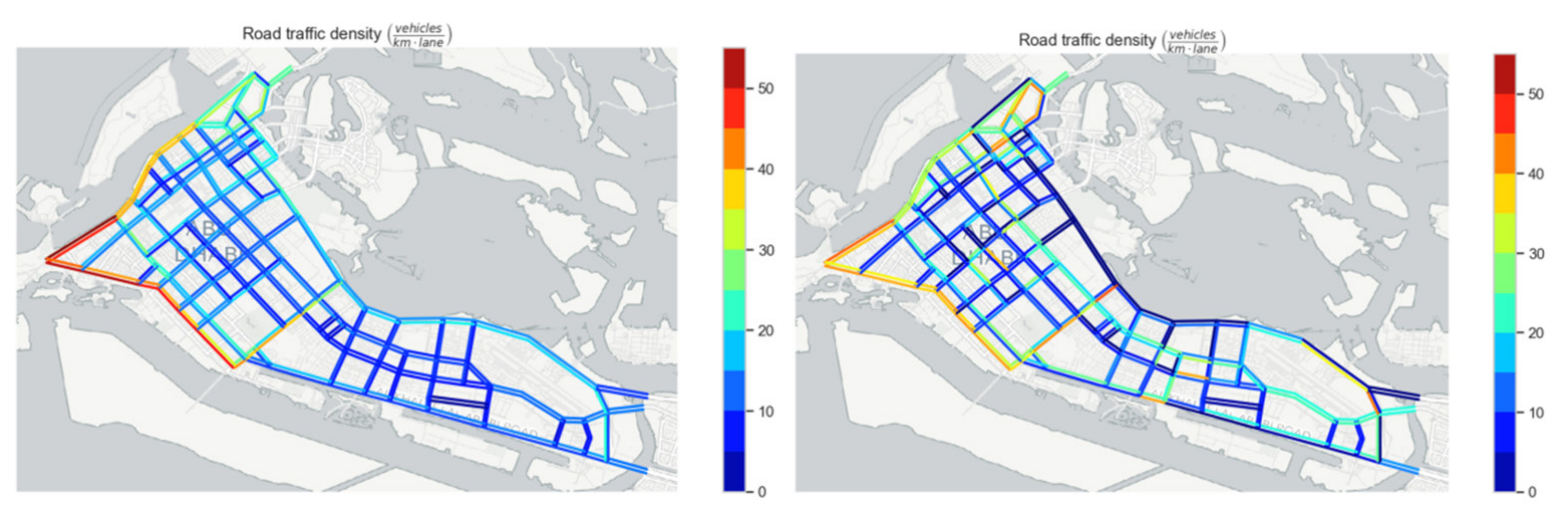

Figure 6. Road traffic density heat map;

left map illustrates the unmodified road network with $D_{\max }=51.23$ vehicles $/ \mathrm{km} \cdot$ lane, right map illustratesthe compromise solution with $D_{\max }=40.99$ vehicles $/ \mathrm{km} \cdot$ lane
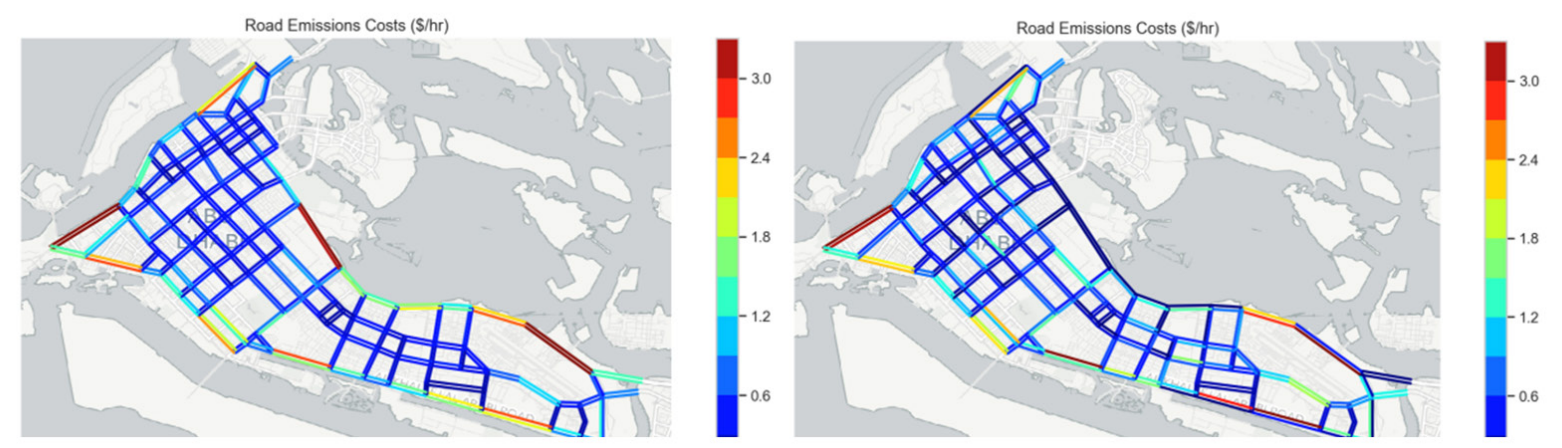

Figure 7. Road emissions cost heat map;

left map illustrates the unmodified road network with TEC $=\$ 213.06 / \mathrm{hr}$, right map illustratesthe compromise solution with TEC $=\$ 189.27 / \mathrm{hr}$ 
Mar. 2000, doi: 10.1016/S1361-9209(99)00031-0.

[5] Y. Wang and W. Y. Szeto, "Multiobjective environmentally sustainable road network design using Pareto optimization," Computer Aided Civil and Infrastructure Engineering, vol. 32, no. 11, pp. 964-987, 2017.

[6] W. Y. Szeto, X. Jaber, and S. C. Wong, "Road Network Equilibrium Approaches to Environmental Sustainability," Transport Reviews, vol. 32, no. 4, pp. 491-518, Jul. 2012, doi: $10.1080 / 01441647.2012 .690000$.

[7] "Fast Facts on Transportation Greenhouse Gas Emissions," US EPA, 25-Aug-2015. [Online]. Available: https://www.epa.gov/greenvehicles/fast-facts-transportation -greenhouse-gas-emissions. [Accessed: 17-Jun-2019].

[8] L. Fabian, N. Cali, and B. Henrik, "Corporate Response to Climate Change Mitigation: What Can We Learn From Annual Reports of European Industries?," International Journal of Industrial Engineering and Management, vol. 2, no. 3, pp. 77-86, 2011.

[9] S. Salman and S. Alaswad, "Alleviating road network congestion: Traffic pattern optimization using Markov chain traffic assignment," Computers \& Operations Research, vol. 99, pp. 191-205, Nov. 2018, doi: 10.1016/j.cor.2018.06.015.

[10] K. Deb, S. Agrawal, A. Pratap, and T. Meyarivan, "A fast elitist non-dominated sorting genetic algorithm for multiobjective optimization: NSGA-II," in International Conference on Parallel Problem Solving From Nature, 2000, pp. 849-858, doi: 10.1007/3-540-45356-3_83.

[11] E. Crisostomi, S. Kirkland, and R. Shorten, "A Google-like model of road network dynamics and its application to regulation and control," International Journal of Control, vol. 84, no. 3, pp. 633-651, Mar. 2011, doi: 10.1080/00207179.2011.568005.

[12] D. A. Levin and Y. Peres, Markov Chains and Mixing Times, second edition, vol. 107. American Mathematical Society, 2017.

[13] Bjorn Klaassen Bos, "Improving traffic mathematically: Ex panding on the work of Crisostomi et al. (2011)," Utrecht University, 2014.

[14] E. Crisostomi, S. Kirkland, A. Schlote, and R. Shorten, "Markov chain based emissions models: A precursor for green control," Green IT: Technologies and Applications, pp. 381-400, 2011.

[15] M. Faizrahnemoon, A. Schlote, L. Maggi, E. Crisostomi, and R. Shorten, "A big-data model for multi-modal public transportation with application to macroscopic control and optimisation," International Journal of Control, vol. 88, no. 11, pp. 2354-2368, Nov. 2015, doi: $10.1080 / 00207179.2015 .1043582$.

[16] S. Salman and S. Alaswad, "Urban road network crisis response management: Time-sensitive decision optimization," in Proceedings of the 2017 Industrial and Systems Engineering Conference, Pittsburgh, PA, 2017, pp. $1307-1313$

[17] X. Zhao, "Modeling Transportation Networks and Urban Traffic Dynamics: A Markovian Framework,” 2017.

[18] Highway capacity manual. Washington, DC: Transportation Research Board, 2000.

[19] R. Smit, "An Examination of Congestion in Road Traffic Emission Models and Their Application to Urban Road Networks," 2006.

[20] M. A. Penic and J. Upchurch, "TRANSYT-7F: enhancement for fuel consumption, pollution emissions, and user costs," Transportation Research Record, no. 1360, 1992.

[21] L. Chen and H. Yang, "Managing congestion and emissions in road networks with tolls and rebates," Transportation Research Part B: Methodological, vol. 46, no. 8, pp. 933-948, Sep. 2012, doi: 10.1016/j.trb.2012.03.001.

[22] J. Long, W. Y. Szeto, and H.-J. Huang, "A bi-objective turning restriction design problem in urban road networks," European Journal of Operational Research, vol. 237, no. 2, pp. 426-439, Sep. 2014, doi: 10.1016/j.ejor.2014.01.053.

[23] R. Ma, X. (Jeff) Ban, and W. Y. Szeto, "Emission Modeling and Pricing in Dynamic Traffic Networks," Transportation Research Procedia, vol. 9, pp. 106-129, 2015, doi: 10.1016/j.trpro.2015.07.007.

[24] W. Y. Szeto, Y. Jiang, D. Z. W. Wang, and A. Sumalee, "A Sustainable Road Network Design Problem with Land Use Transportation Interaction over Time," Networks and Spatial Economics, vol. 15, no. 3, pp. 791-822, Sep. 2015, doi: 10.1007/s11067-013-9191-9.

[25] Y. Yin and S. Lawphongpanich, "Internalizing emission externality on road networks," Transportation Research Part D: Transport and Environment, vol. 11, no. 4, pp. 292-301, Jul. 2006, doi: 10.1016/j.trd.2006.05.003.

[26] H. S. Matthews, "The External Costs of Air Pollution and the Environmental Impact of the Consumer in the U.S. Economy,” p. 203, 1999.

[27] W. Y. Szeto, Y. Wang, and S. C. Wong, "The Chemical Reaction Optimization Approach to Solving the Environmentally Sustainable Network Design Problem,” Computer-Aided Civil and Infrastructure Engineering, vol. 29, no. 2, pp. 140-158, Feb. 2014, doi: 10.1111/mice.12033.

[28] A. Konak, D. W. Coit, and A. E. Smith, "Multi-objective optimization using genetic algorithms: A tutorial," Reliability Engineering \& System Safety, vol. 91, no. 9, pp. 992-1007, Sep. 2006, doi: 10.1016/j.ress.2005.11.018.

[29] F.-A. Fortin, F.-M. D. Rainville, M.-A. Gardner, M. Parizeau, and C. Gagné, "DEAP: Evolutionary Algorithms Made Easy," Journal of Machine Learning Research, vol. 13, pp. 2171-2175, Jul. 2012.

[30] E. Jones, T. Oliphant, P. Peterson, and others, SciPy: Open source scientific tools for Python. 2001. 